\title{
Autoeficacia Percibida en la conducta Académica de Estudiantes Universitarias
}

\author{
Martha Ornelas, Humberto Blanco, Gabriel Gastélum y Alejandro Chávez \\ Universidad Autónoma de Chihuahua. Facultad de Educación Física y Ciencias del Deporte. DES \\ Salud UACH-CAs 121, 101 y 26. Calle Escorza 900, CP 31000 Chihuahua, Chih. México. \\ (e-mail: mornelas@uach.mx, hblanco@uach.mx,gastelum@uach.mx y achavezg@uach.mx)
}

Recibido Sep. 05, 2011; Aceptado Nov. 02, 2011; Versión final recibida Ene. 24, 2011

\begin{abstract}
Resumen
Se ha realizado un estudio para caracterizar a las estudiantes que optan por alguna de las licenciaturas que se ofrecen en la Universidad Autónoma de Chihuahua en México, en cuanto a la autoeficacia percibida en su desempeño académico. La muestra consistió de 902 mujeres estudiantes de primer ingreso de siete disciplinas, con una edad media de 18.1 años. El método de investigación se enmarcó dentro de un enfoque cuantitativo con un diseño descriptivo tipo encuesta. Los resultados muestran que los perfiles de autoeficacia percibida son muy similares entre las alumnas de las diferentes disciplinas, y que las conductas relacionadas con el factor comunicación representan un área de oportunidad para las alumnas de nuevo ingreso. Además, se observó que los perfiles de autoeficacia actual, deseada y alcanzable están relacionadas: a mayor autoeficacia percibida, mayor deseo y mayores posibilidades de ser eficaz. Esto permite concluir que si se logra mejorar cualquiera de ellos los otros dos también lo harán.
\end{abstract}

Palabras clave: autoeficacia, conducta académica, desempeño académico, mujeres estudiantes

\section{Perceived self-efficacy in the academic behavior of female university students}

\begin{abstract}
A study has been done to characterize female students who choose one of the degrees offered by the Universidad Autónoma de Chihuahua in Mexico, in terms of the perceived self-efficacy in their academic performance. The sample consisted of 902 female freshmen college students from seven disciplines with a mean age of 18.1 years. The research method adopted has a quantitative focus with a descriptive design employing a survey. The results show that the profiles of selfefficacy in academic behavior is very similar between students of different disciplines, and that the behavior related to communication skills represents an area of opportunity for improvement in incoming college students. Also, it was observed that the profiles of current, desired and achievable efficacy are related: the higher the perceived self-efficacy, greater desire and more chances of being effective. This allows concluding that if any of them can be improved, the other two will also do it.
\end{abstract}

Keywords: self-efficacy, academic behavior, academic performance, female students 


\section{INTRODUCCIÓN}

La autoeficacia es tradicionalmente entendida como referida a un dominio o una tarea específica. Sin embargo, algunos investigadores también han conceptualizado un sentido general de autoeficacia que se refiere a un sentido amplio y estable de competencia personal sobre cuán efectiva puede ser la persona al afrontar una variedad de situaciones estresantes (Choi, 2004; Luszczynska et al., 2004; Luszczynska et al., 2005). Así, resulta posible definir el término autoeficacia como los juicios que cada individuo hace sobre sus capacidades, en base a los cuales organizará y ejecutará sus actos de modo que le permitan alcanzar el rendimiento deseado o como las creencias que tiene la persona sobre sus capacidades para organizar y ejecutar caminos para la acción requeridos en situaciones esperadas o basadas en niveles de rendimiento (Ornelas et al., en prensa). Bandura (2001) plantea que la autoeficacia percibida debe ser conceptualizada de manera específica. La autoeficacia percibida hace pues referencia a las creencias de las personas acerca de sus propias capacidades para el logro de determinados resultados. Por consiguiente, el sistema de creencias de eficacia no es un rasgo global sino un grupo de auto-creencias ligadas a ámbitos de funcionamiento diferenciado.

La teoría social-cognitiva desarrollada por Bandura enfatiza el papel de los fenómenos autorrefenciales como el medio por el cual el ser humano es capaz de actuar en su ambiente y por consecuencia transformarlo, las personas crean y desarrollan autopercepciones acerca de su capacidad, mismas que se convierten en los medios por los cuales siguen sus metas y toman sus decisiones (Gutiérrez et al., 2011; Sansinenea et al., 2008). Esto es, la forma en que la gente actúa es en parte producto de la intervención de sus creencias acerca de lo que es capaz de realizar.

Dentro de los contextos educativos ha existido un interés permanente por comprender los factores cognitivos y comportamentales que favorecen o dificultan el desempeño del estudiante en sus labores académicas y cómo éste se relaciona con su desarrollo integral. En el área de la psicología educativa específicamente, el constructo de autoeficacia ha recibido especial atención y se han generado importantes avances de investigación que han contribuido al mejoramiento de prácticas pedagógicas y de enseñanza (Pérez et al., 2011). La investigación empírica ha demostrado ampliamente que la autoeficacia resulta ser más predictiva del rendimiento académico que otras variables cognitivas (Brown et al., 2008; Pérez et al., 2011; Schmidt et al. 2008), también que logra predecir el éxito posterior (Pajares y Schunk, 2001; Pérez et al., 2005) y que es un importante mediador cognitivo de competencia y rendimiento (Valiante, 2000; Vera et al., 2011) en cuanto favorece los procesos cognoscitivos.

La autoeficacia percibida pues, desempeña un papel fundamental en el funcionamiento humano puesto que, afecta el comportamiento no sólo de manera directa, sino también por su impacto en otros determinantes claves tales como metas y aspiraciones, expectativas de resultados, tendencias afectivas y percepción de los impedimentos y oportunidades que se presentan en el medio social (Bandura, 1997; García et al., 2007).

Con lo dicho hasta aquí se ha querido hacer hincapié en la posibilidad que tienen las creencias de autoeficacia de ser desarrolladas y con ello incrementar la oportunidad de las personas para obtener mejores desempeños. Tratando siempre de afianzar la idea de que darse a la tarea de mejorar la percepción de ser capaz en quien aprende es un objetivo educacional valioso, bajo el supuesto implícito de que su potenciación servirá como vehículo para la mejora de otros resultados tales como el logro académico y la autoestima. Tomando en cuenta que la continua conciencia de fracaso reduce las expectativas de éxito y no favorece en ningún modo ni el aprendizaje ni el desarrollo personal.

Por todo ello, esta investigación es fundamentalmente un estudio de tipo descriptivo que intenta caracterizar a las estudiantes que optan por alguna de las licenciaturas de la UACH, en cuanto a la eficacia percibida en su desempeño académico, al comparar su perfil con el de las alumnas que eligen otro tipo de carrera. El presente estudio contribuye pues a aportar evidencias y datos que propician la intervención educativa dentro de una perspectiva educativa de atención a la diversidad en el aula. 


\section{METODOLOGÍA}

\section{Sujetos}

Participaron en esta investigación un total de 902 estudiantes de 17 a 20 años, todas alumnas de primer grado de las licenciaturas que se ofrecen en la $\mathrm{UACH}$; se utilizó un muestreo por conveniencia, tratando de abarcar la representatividad de las diferentes licenciaturas (Tabla 1).

Tabla 1: Distribución de los sujetos de acuerdo a las variables disciplina y edad.

\begin{tabular}{lcc}
\hline & \multicolumn{2}{c}{ Edad } \\
\cline { 2 - 3 } \multicolumn{1}{c}{ Disciplina } & $\mathrm{n}$ & $\mathrm{M}(\mathrm{DE})$ \\
\hline Educación y Humanidades & 96 & $18.25(.79)$ \\
Ciencias de la Salud & 121 & $18.12(.76)$ \\
Educación Física & 81 & $18.14(.67)$ \\
Ciencias Agropecuarias & 85 & $18.32(.88)$ \\
Ciencias Políticas & 200 & $18.16(.72)$ \\
Ciencias Sociales y Administrativas & 176 & $18.10(.61)$ \\
Ingeniería y Tecnología & 143 & $18.02(.65)$ \\
Total & 902 & $18.14(.72)$ \\
\hline
\end{tabular}

\section{Instrumento}

Escala Autoeficacia en Conductas Académicas (EACA) Encuesta tipo Likert, asistida por computadora, de 13 ítems relacionados con conductas académicas; donde el encuestado responde, en una escala de 0 a 10, la frecuencia con que actualmente, en forma ideal y si se esfuerza en cambiar, realizaría o manifestaría una acción. Para luego a partir de sus respuestas obtener 5 índices: 1) Autoeficacia percibida actualmente.- obtenida a partir de las respuestas al escenario actual. 2) Autoeficacia deseada.- obtenida a partir de las respuestas al escenario ideal. 3) Autoeficacia alcanzable en el futuro.- obtenida a partir de las respuestas al escenario de cambio. 4) Grado de insatisfacción o disonancia en la autoeficacia percibida.- obtenida a través de la diferencia entre el índice 2 y 1 (ideal menos actual). Y 5) Posibilidad de mejoría en la autoeficacia percibida.- obtenida a través de la diferencia entre el índice 3 y 1 (cambio menos actual).

La estructura de tres factores: Comunicación, Atención y Excelencia, para esta escala (Tabla 2), atendiendo a criterios estadísticos y sustantivos, ha mostrado adecuados indicadores de ajuste, de fiabilidad y de validez (Blanco et al., 2011a; Blanco et al., 2011b). Se eligió este tipo de encuesta por ser fácil de construir y de aplicar; además, de proporcionar una buena base para una primera ordenación de los individuos en la característica que se mide (Raviolo et al., 2010).

\section{Variable explicativa}

Disciplina. Con los siguientes valores: Educación y Humanidades; Ciencias de la Salud; Educación Física; Ciencias Agropecuarias; Ciencias políticas; Ciencias Sociales y Administrativas; Ingeniería y Tecnología. 
Tabla 2: Ítems de Escala Autoeficacia en Conductas Académicas, agrupados por factor.

\begin{tabular}{lll}
\hline Factor & & \multicolumn{1}{c}{ Ítem } \\
\hline Comunicación & 4 Expreso mis ideas con claridad \\
& 5 Hago comentarios y aportaciones pertinentes \\
& 12 En caso de desacuerdo soy capaz de entablar un diálogo con mis profesores \\
& 13 Me siento bien con mi propio desempeño cuando hablo enfrente de una \\
& 2 clase o grupo de gente \\
Atención & 3 Escucho con atención cuando el profesor aclara una duda a un compañero \\
& 6 Pongo atención cuando los profesores dan la clase \\
& 7 Pongo atención cuando un compañero expone en clase \\
& 11 Escucho con atención las preguntas y comentarios de mis profesores \\
Excelencia & 1 Cumplo con las tareas que se me asignan \\
& 8 Me preparo para mis exámenes apoyándome en los apuntes de clase, el \\
& 9 texto del curso y lecturas adicionales \\
& 10 Entrego puntualmente los trabajos que se me encargan \\
&
\end{tabular}

Variables de respuesta

Puntaje de los cinco índices antes descritos en cada uno de tres factores: Comunicación, Atención y Excelencia

\section{Procedimiento}

Se invitó a participar en el estudio a las estudiantes de primer ingreso de las licenciaturas que se ofrecen en la $\mathrm{UACH}$; las que aceptaron participar firmaron la carta de aceptación correspondiente. Luego se aplicó el instrumento antes descrito por medio de una computadora personal utilizando el módulo administrador del instrumento del editor de escalas versión 2.0 (Blanco et al., 2007), en una sesión de aproximadamente 25 minutos; en los centros de cómputo de las unidades académicas participantes. Al inicio de cada sesión se hizo una pequeña introducción sobre la importancia de la investigación y de cómo acceder al instrumento; las instrucciones de como responder se encontraban en las primeras pantallas; antes del primer reactivo del instrumento. Al término de la sesión se les agradeció su participación. Una vez aplicado el instrumento se procedió a recopilar los resultados por medio del módulo generador de resultados del editor de escalas versión 2.0 (Blanco et al., 2007).

\section{Análisis de datos}

Para comparar los resultados obtenidos por las distintas disciplinas se utilizó el análisis de varianza entre grupos, tras comprobar que los datos cumplían el criterio de normalidad estadística. Y para los perfiles de autoeficacia de los factores estudiados se utilizó el análisis de varianza de medidas repetidas. En ambos casos se aplicó el test de Scheffé para comprobar las diferencias entre pares.

\section{RESULTADOS Y DISCUSIÓN}

\section{Factor Comunicación}

De acuerdo a los resultados obtenidos existen diferencias significativas en dos de los cinco índices estudiados (Tabla 3). En el factor Comunicación, las alumnas de Educación Física y las de Ciencias Políticas se perciben con un menor nivel de autoeficacia deseada que las de Ciencias 
Sociales y Administrativas; y las de Ciencias de la Salud manifiestan mayor insatisfacción o disonancia en la autoeficacia percibida que las de Educación Física (Tabla 4). En la Tabla 4 la notación es: Nota. $\mathrm{EH}=$ Educación y Humanidades; $\mathrm{CS}=$ Ciencias de la Salud; EF = Educación Física; $\mathrm{CA}=$ Ciencias Agropecuarias; $\mathrm{CP}=$ Ciencias Políticas; $\mathrm{CSA}=$ Ciencias Sociales $\mathrm{y}$ Administrativas; IT = Ingeniería y Tecnología. Las medias en la misma fila que comparten el mismo subíndice difieren entre sí a un nivel de cuando menos $p=.05$

Tabla 3: Análisis de varianza para el factor Comunicación $(* p<.05 \quad * * p<.01 \quad$ *** $p<.005)$

\begin{tabular}{lcccc}
\hline \multicolumn{1}{c}{ Fuente } & $S C$ & $g l$ & $M C$ & $F$ \\
\hline \multicolumn{5}{c}{ Autoeficacia percibida actualmente } \\
Disciplina & 25.58 & 6 & 4.26 & 1.82 \\
Error & 2098.54 & 895 & 2.34 \\
\hline \multicolumn{5}{c}{ Autoeficacia deseada } \\
Disciplina & 23.08 & 6 & 3.85 & $4.54^{\star \star \star}$ \\
Error & 757.90 & 895 & 0.85 \\
\hline \multicolumn{5}{c}{} \\
Disciplina & Autoeficacia alcanzable en el futuro \\
Error & 9.29 & 6 & 1.55 & 2.07 \\
\hline Grado de insatisfacción o disonancia en la autoeficacia percibida \\
Disciplina & 20.42 & 6 & 3.40 \\
Error & 1383.73 & 895 & $2.20^{*}$ \\
\hline \multicolumn{5}{c}{1.55} \\
Disciplina & Posibilidad de mejoría en la autoeficacia percibida \\
Error & 10.64 & 6 & 1.77 \\
\hline \multicolumn{5}{c}{} \\
\hline
\end{tabular}

Tabla 4: Puntuaciones medias en cada uno de los índices definidos para el factor Comunicación.

\begin{tabular}{|c|c|c|c|c|c|c|c|}
\hline \multirow[b]{2}{*}{ Índice } & \multicolumn{7}{|c|}{ Disciplina } \\
\hline & $\mathrm{EH}$ & CS & EF & CA & $\mathrm{CP}$ & CSA & IT \\
\hline Autoeficacia percibida actualmente & 7.48 & 7.43 & 7.55 & 7.63 & 7.47 & 7.84 & 7.32 \\
\hline Autoeficacia deseada & 9.15 & 9.40 & $8.97 \mathrm{a}$ & 9.22 & $9.09_{b}$ & $9.44_{a b}$ & 9.12 \\
\hline Autoeficacia alcanzable en el futuro & 9.18 & 9.29 & 9.24 & 9.35 & 9.35 & 9.42 & 9.13 \\
\hline $\begin{array}{l}\text { Grado de insatisfacción o disonancia } \\
\text { en la autoeficacia percibida }\end{array}$ & 1.67 & $1.98_{a}$ & $1.42_{\mathrm{a}}$ & 1.59 & 1.61 & 1.60 & 1.79 \\
\hline $\begin{array}{l}\text { Posibilidad de mejoría en la } \\
\text { autoeficacia percibida }\end{array}$ & 1.70 & 1.86 & 1.69 & 1.72 & 1.88 & 1.58 & 1.80 \\
\hline
\end{tabular}

\section{Factor Atención}

De acuerdo a los resultados obtenidos existen diferencias significativas en tres de los cinco índices estudiados (Tabla 5). En el factor Atención, las alumnas de Ciencias Políticas se perciben con menor autoeficacia y mayor posibilidad de mejoría en su autoeficacia que las alumnas de Ciencias de la Salud, de Ciencias Agropecuarias y de Ciencias Sociales y Administrativas; y con menor nivel de autoeficacia deseada que las alumnas de Ingeniería y Tecnología, además de las alumnas de las disciplinas antes mencionadas. Por su parte las alumnas de Ciencias de la Salud 
manifiestan un mayor nivel de autoeficacia deseada que las de Educación Física (Tabla 6). En la Tabla 6 la notación es: $\mathrm{EH}=$ Educación y Humanidades; $\mathrm{CS}=$ Ciencias de la Salud; $\mathrm{EF}=$ Educación Física; $\mathrm{CA}=$ Ciencias Agropecuarias; $\mathrm{CP}=$ Ciencias Políticas; $\mathrm{CSA}=$ Ciencias Sociales y Administrativas; IT = Ingeniería y Tecnología. Las medias en la misma fila que comparten el mismo subíndice difieren entre sí a un nivel de cuando menos $p=.05$

\begin{tabular}{|c|c|c|c|c|}
\hline Fuente & SC & $g l$ & $M C$ & $F$ \\
\hline \multicolumn{5}{|c|}{ Autoeficacia percibida actualmente } \\
\hline Disciplina & 29.69 & 6 & 4.95 & $4.61^{\star \star \star}$ \\
\hline Error & 961.09 & 895 & 1.07 & \\
\hline \multicolumn{5}{|c|}{ Autoeficacia deseada } \\
\hline Disciplina & 26.83 & 6 & 4.47 & $9.80^{* * *}$ \\
\hline Error & 408.51 & 895 & 0.46 & \\
\hline \multicolumn{5}{|c|}{ Autoeficacia alcanzable en el futuro } \\
\hline Disciplina & 2.23 & 6 & 0.37 & 1.35 \\
\hline Error & 246.13 & 895 & 0.28 & \\
\hline
\end{tabular}

Grado de insatisfacción o disonancia en la autoeficacia percibida

\begin{tabular}{lrrrr} 
Disciplina & 5.23 & 6 & 0.87 & 1.46 \\
Error & 534.32 & 895 & 0.60 & \\
\hline
\end{tabular}

Posibilidad de mejoría en la autoeficacia percibida

\begin{tabular}{lrrrr} 
Disciplina & 18.17 & 6 & 3.03 & $4.68^{\star \star *}$ \\
Error & 579.29 & 895 & 0.65 & \\
\hline
\end{tabular}

Tabla 6: Puntuaciones medias en cada uno de los índices definidos para el factor Atención.

\begin{tabular}{|c|c|c|c|c|c|c|c|}
\hline \multirow[b]{2}{*}{ Índice } & \multicolumn{7}{|c|}{ Disciplina } \\
\hline & $\mathrm{EH}$ & CS & $\mathrm{EF}$ & CA & $\mathrm{CP}$ & CSA & IT \\
\hline Autoeficacia percibida actualmente & 8.24 & $8.46_{a}$ & 8.35 & $8.44_{b}$ & $7.96_{a b c}$ & $8.41_{c}$ & 8.27 \\
\hline Autoeficacia deseada & 9.44 & $9.71_{\mathrm{ab}}$ & $9.32_{a}$ & $9.50_{c}$ & $9.16_{\text {bcde }}$ & $9.52_{d}$ & $9.45_{\mathrm{e}}$ \\
\hline Autoeficacia alcanzable en el futuro & 9.55 & 9.67 & 9.55 & 9.64 & 9.54 & 9.64 & 9.58 \\
\hline $\begin{array}{l}\text { Grado de insatisfacción o disonancia } \\
\text { en la autoeficacia percibida }\end{array}$ & 1.19 & 1.24 & 0.98 & 1.06 & 1.20 & 1.12 & 1.18 \\
\hline $\begin{array}{l}\text { Posibilidad de mejoría en la } \\
\text { autoeficacia percibida }\end{array}$ & 1.30 & $1.21_{\mathrm{a}}$ & 1.21 & $1.19_{b}$ & $1.57 \mathrm{abc}$ & $1.23_{\mathrm{c}}$ & 1.32 \\
\hline
\end{tabular}

\section{Factor Excelencia}

De acuerdo a los resultados obtenidos existen diferencias significativas en tres de los cinco índices estudiados (Tabla 7). En el factor Excelencia, las alumnas de Educación y Humanidades se perciben con menor autoeficacia y menos deseos de ser autoeficaces; pero con mayor posibilidad de mejoría en su autoeficacia; que las alumnas de Ciencias de la Salud; y las de Ciencias de la salud con mayor nivel de autoeficacia deseada que las alumnas de las disciplinas de Educación Física y Ciencias Políticas; a su vez estas últimas con menos autoeficacia deseada que las de Ciencias Sociales y Administrativas (Tabla 8). En la Tabla 8 la notación es: Nota. EH = Educación y Humanidades; CS = Ciencias de la Salud; EF = Educación Física; CA = Ciencias Agropecuarias; $\mathrm{CP}=$ Ciencias Políticas; $\mathrm{CSA}=$ Ciencias Sociales y Administrativas; $\mathrm{IT}=$ 
Ingeniería y Tecnología. Las medias en la misma fila que comparten el mismo subíndice difieren entre sí a un nivel de cuando menos $p=.05$.

Tabla 7: Análisis de varianza para el factor Excelencia.

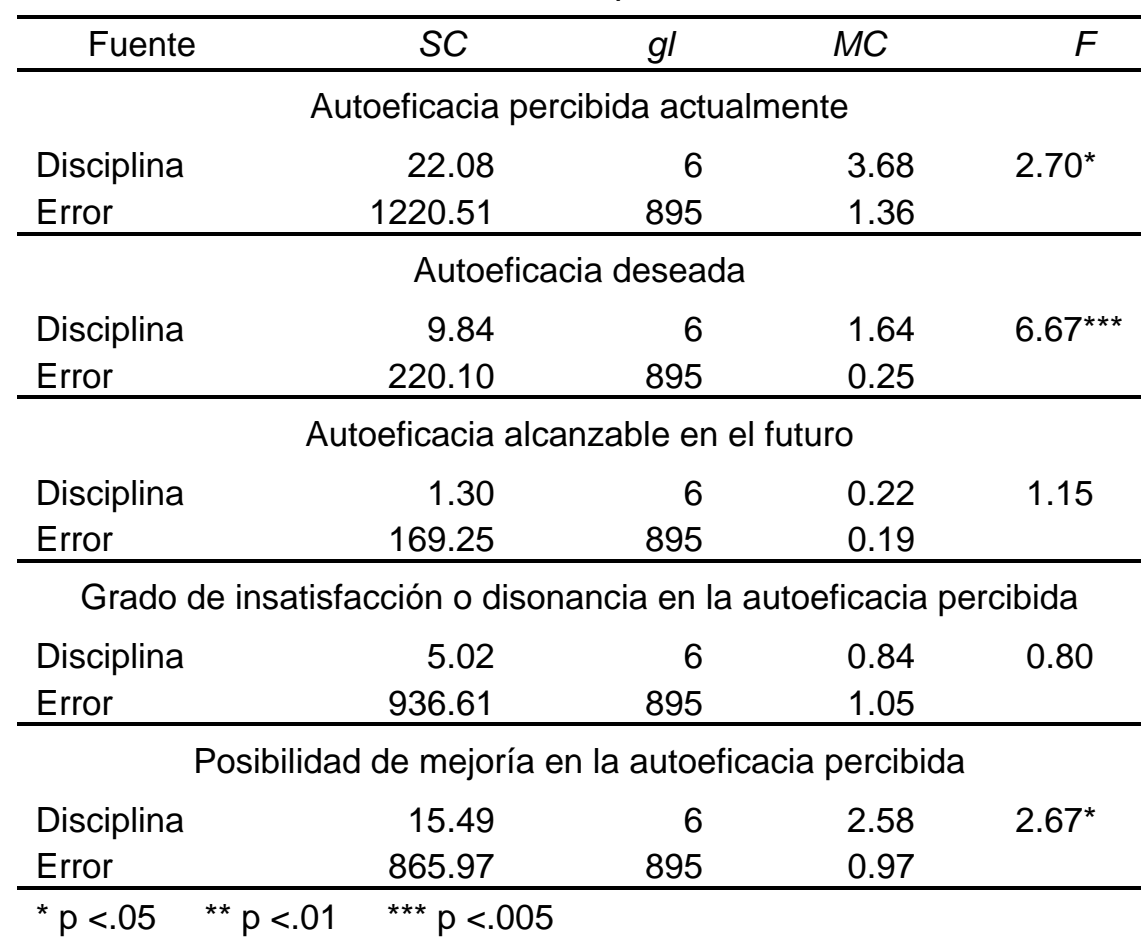

Tabla 8: Puntuaciones medias en cada uno de los índices definidos para el factor Excelencia.

\begin{tabular}{lcccccccc}
\hline & \multicolumn{7}{c}{ Disciplina } \\
\cline { 2 - 8 } \multicolumn{1}{c}{ Índice } & EH & CS & EF & CA & CP & CSA & IT \\
\hline Autoeficacia percibida actualmente & $8.27_{\mathrm{a}}$ & $8.82_{\mathrm{a}}$ & 8.47 & 8.56 & 8.43 & 8.65 & 8.60 \\
Autoeficacia deseada & $9.62_{\mathrm{a}}$ & $9.89_{\mathrm{abc}}$ & $9.60_{\mathrm{b}}$ & 9.69 & $9.58_{\mathrm{cd}}$ & $9.76_{\mathrm{d}}$ & 9.73 \\
Autoeficacia alcanzable en el futuro & 9.64 & 9.77 & 9.73 & 9.76 & 9.76 & 9.76 & 9.77 \\
$\begin{array}{l}\text { Grado de insatisfacción o disonancia } \\
\text { en la autoeficacia percibida }\end{array}$ & 1.35 & 1.08 & 1.13 & 1.12 & 1.15 & 1.11 & 1.13 \\
$\begin{array}{l}\text { Posibilidad de mejoría en la } \\
\text { autoeficacia percibida }\end{array}$ & $1.38_{\mathrm{a}}$ & $0.95_{\mathrm{a}}$ & 1.26 & 1.20 & 1.33 & 1.11 & 1.17 \\
\hline
\end{tabular}

\section{Factores Comunicación-Atención-Excelencia}

Los resultados de los análisis de varianza de medidas repetidas para autoeficacia percibida actualmente $F(2,1802)=265.807, p<.005$; autoeficacia deseada $F(2,1802)=231.814, p<.005$ y para autoeficacia alcanzable en el futuro $F(2,1802)=244.855, p<.005$ muestran que Comunicación es el factor donde las alumnas universitarias se perciben con menos autoeficacia, con menos deseos y posibilidades de ser autoeficaces; mientras que Excelencia es el factor donde se perciben actualmente con mayor autoeficacia, deseos y posibilidades de ser autoeficaces (Figura 1). También es importante destacar que los perfiles de autoeficacia actual, deseada y alcanzable se corresponden, estos es: entre mayor autoeficacia percibida, mayor deseo y mayores posibilidades de ser eficaz. 


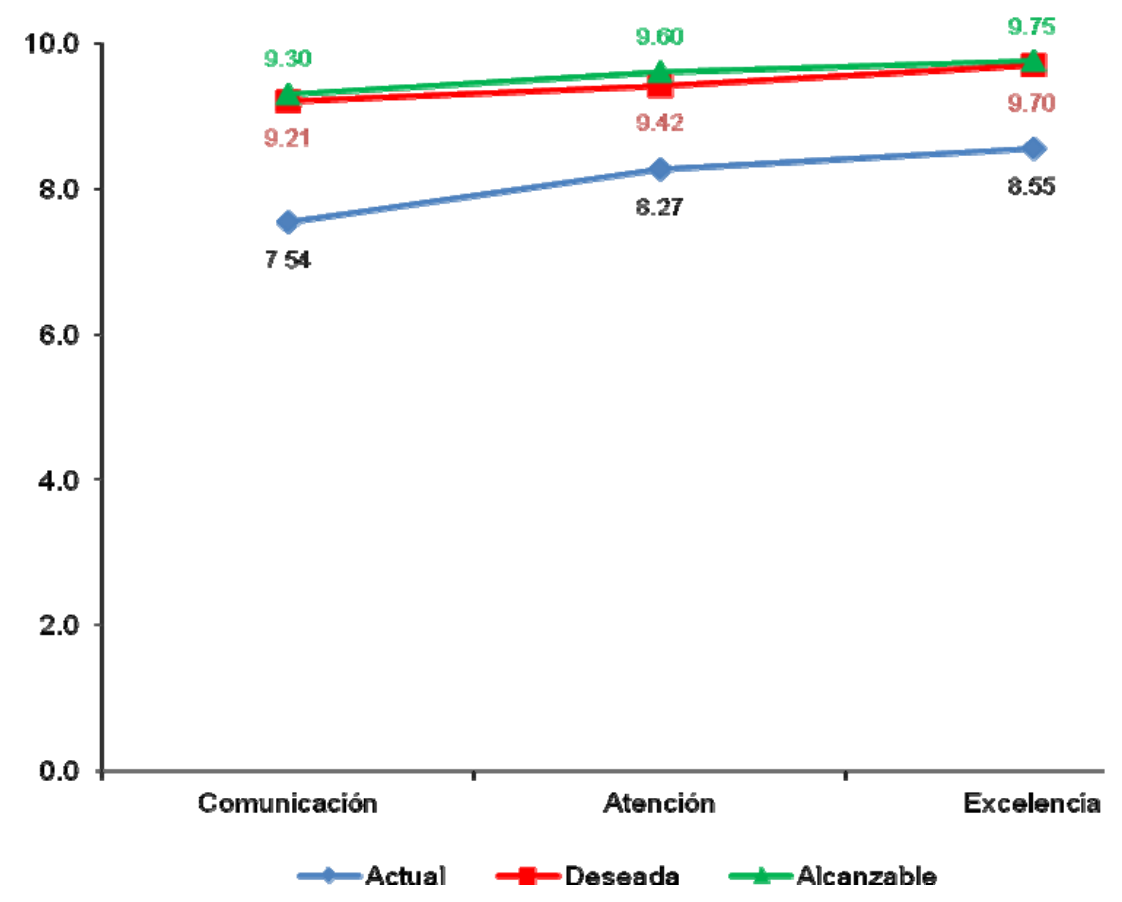

Fig. 1 Perfiles de autoeficacia actual, deseada y alcanzable en el futuro en cada uno de los factores estudiados.

\section{CONCLUSIONES}

De los resultados mostrados, de su análisis y de su discusión, se pueden obtener las siguientes conclusiones:

1) en cuanto a las conductas académicas estudiadas, se puede afirmar que las alumnas sin importar la disciplina a la cual pertenece la licenciatura que estudian se perciben tan autoeficaces y con la misma posibilidad de mejoría en su autoeficacia que las alumnas de las demás disciplinas;

2) las alumnas de nuevo ingreso a la universidad, en general, tienden a sentirse menos eficaces, con menor necesidad y posibilidad de ser más autoeficaces en lo que respecta al factor comunicación, esto en cuanto a expresar ideas con claridad, hacer comentarios y aportaciones pertinentes, en caso de desacuerdo ser capaz de entablar un diálogo con los profesores, sentirse bien con su desempeño cuando se habla enfrente de una clase o grupo de gente;

3) el que los perfiles de autoeficacia actual, deseada y alcanzable se correspondan (a mayor autoeficacia percibida, mayor deseo y mayores posibilidades de ser eficaz) permite concluir que si se logra mejorar cualquiera de ellos los otros dos también lo harán; y

4) la disonancia cognoscitiva generada en relación a la autoeficacia percibida en el presente, el nivel de autoeficacia deseado o ideal, y la posibilidad de mejoría en la autoeficacia percibida; puede ser aprovechada por el docente o tutor académico de la estudiante para que ésta establezca metas de logro para aumentar su eficacia, aproximándola a la deseada o alcanzable.

\section{REFERENCIAS}

Bandura, A., Self-efficacy: The exercise of Control, Freeman, New York (1997).

Bandura, A., Guía para la construcción de escalas de autoeficacia (2001). http://www.revistaevaluar.com.ar/effguideSpanish.htm. Acceso: 16 mayo (2011) 
Blanco, H., Martínez, M. A., Ornelas, M., Flores, F. J. y Peinado, J. E., Validación de las escalas autoeficacia en conductas académicas y cuidado de la salud, Doble Hélice Ediciones, México (2011a).

Blanco, H., Martínez, M. A., Zueck, M. C. y Gastélum G., Análisis psicométrico de la escala autoeficacia en conductas académicas en universitarios de primer ingreso, Revista Actualidades Investigativas en Educación, 11(3), 1-27 (2011b)

Blanco, H. y otros siete autores, Editor para la construcción y aplicación de escalas por medio de una PC, Tecnociencia Chihuahua, 1(1), 55-59 (2007).

Brown, S. D., Tramayne, S., Hoxha, D., Telander, K., Fan, X. y Lent, R. W., Social cognitive predictors of college students'academic performance and persistence: a meta-analytic path analysis, Journal of Vocational Behavior, ISSN: 0001-8791 (en línea), 72 (3), 298-308, 2008. http://dx.doi.org/10.1016/j.jvb.2007.09.003. Acceso: 3 de agosto (2011).

Choi, N., Sex role group differences in specific, academic, and general self-efficacy. The Journal of Psychology, 138, 149-159 (2004).

García, A. L., García, M. y Ramos, P. J., Aportaciones de la inteligencia emocional y la autoeficacia: Aplicaciones para la selección de personal, Anales de Psicología, 23(2), 231-239, (2007).

Gutiérrez, M., Escartí, A. y Pascual, C., Relaciones entre empatía, conducta prosocial, agresividad, autoeficacia y responsabilidad personal y social de los escolares, Psicothema, ISSN: 1886-144X (en línea), 23(1), 13-19, 2011. www.psicothema.com/pdf/3843.pdf. Acceso: 3 de agosto (2011).

Luszczynska, A., Gibbons, F., Piko, B. y Tekozel, M., Self-regulatory cognitions, social comparison, perceived peers' behaviors as predictors of nutrition and physical activity: $A$ comparison among adolescents in Hungary, Poland, Turkey, and USA. Psychology and Health, 19, 577-593 (2004).

Luszczynska, A., Scholz, U. y Schwarzer, R., The general self-efficacy scale: Multicultural validation studies, The Journal of Psychology, ISSN: 0022-3980, 139 (5), 439-457, 2005. http://userpage.fu-berlin.de/ health/self/gse-multicult_2005.pdf. Acceso: 4 de agosto (2011).

Ornelas, M., Blanco, H., Rodríguez, J. M. y Flores, F. J., Análisis Psicométrico de la Escala Autoeficacia en Conductas de Cuidado de la Salud Física en Universitarios de Primer Ingreso, Form. Univ., 4(6), en prensa (2011).

Pajares, F., y Schunk, D. H., Self-Beliefs and School Success: Self-Efficacy, Self-Concept, and School Achievement. In R. Riding y S. Rayner (Eds.), Perception, pp. 239-266 Ablex Publishing, London (2001).

Pérez, E., Cupani, M. y Ayllón, S., Predictores de rendimiento académico en la escuela media: aptitudes, autoeficacia y rasgos de personalidad, Avaliaçao Psicológica, ISSN: 1677-0471 (en línea), 4(1), 1-11, 2005. http://pepsic.bvsalud.org/pdf/avp/v4n1/v4n1a02.pdf. Acceso: 3 de agosto (2011).

Pérez, E., Lescano, C., Zalazar, P., Furlám, L. y Martínez, M., Desarrollo y análisis psicométricos de un Inventario de Autoeficacia para Inteligencias Múltiples en Niños Argentinos. Psicoperspectivas, ISSN: 0718-6924 (en línea), 10 (1), 169-189, 2011. http://www.psicoperspectivas.cl/index.php/psicoperspectivas/article/viewFile/141/129. Acceso: 3 de agosto (2011). 
Raviolo, A., Ramirez, P., López, E. A. y Aguilar, A., Concepciones sobre el Conocimiento y los Modelos Científicos: Un Estudio Preliminar, Form. Univ., 3(5), 29-36, (2010).

Sansinenea, y otros seis autores, Autoconcordancia y autoeficacia en los objetivos personales: ¿Cuál es su aportación al bienestar?, Anales de Psicología, 24(1), 121-128, (2008).

Schmidt, V., Messoulam, N. y Molina, F., Autoconcepto académico en adolescentes de escuelas medias: presentación de un instrumento para su evaluación, Revista Iberoamericana de Diagnóstico y Evaluación Psicológica, 1(25), 81-106, (2008).

Valiante, G., Writing Self-efficacy and gender orientation: A developmental perspective, a dissertation proposal. Emory University, Atlanta (2000).

Vera, M., Salanova, M. y Martín-del-Río, B., Self-efficacy among university faculty: how to develop an adjusted scale, Anales de Psicología, 27(3), 800-807, (2011). 